\title{
Acuidade visual e função de bastonetes em pacientes com retinose pigmentária
}

\author{
Visual acuity and rod function in patients with retinitis pigmentosa
}

\author{
Adriana Berezovsky ${ }^{1}$ \\ Josenilson Martins Pereira ${ }^{2}$ \\ Paula Yuri Sacai ${ }^{2}$ \\ Sérg'io Costa Fantini ${ }^{3}$ \\ Solange Rios Salomão ${ }^{4}$
}

Trabalho realizado no Laboratório de Eletrofisiologia Visual Clínica do Departamento de Oftalmologia da Universidade Federal de São Paulo - UNIFESP.

${ }^{1}$ Professora Adjunta Doutora de Departamento de Oftalmologia da Universidade Federal de São Paulo UNIFESP.

2 Tecnólogo(a) Oftálmico(a) de Departamento de Oftalmologia da Universidade Federal de São Paulo - UNIFESP.

${ }^{3}$ Médico Oftalmologista, Colaborador Voluntário de Setor de Eletrofisiologia Visual Clínica do Departamento de Oftalmologia da Universidade Federal de São Paulo - UNIFESP.

${ }^{4}$ Professora Adjunta Doutora, Chefe do Laboratório de Eletrofisiologia Visual Clínica do Departamento de Oftalmologia da Universidade Federal de São Paulo UNIFESP.

Endereço para Correspondência: Adriana Berezovsky, R. Botucatu, 822 - São Paulo (SP) - CEP 04023-062

E-mail: aberezovsky@oftalmo.epm.br

Nota Editorial: Pela análise deste trabalho e por sua anuência na divulgação desta nota, agradecemos ao Dr. Sérgio Lustosa da Cunha.

Recebido para publicação em 29.05.2003

Versão revisada recebida em 23.09.2003

Aprovação em 10.01.2004

\begin{tabular}{|c|}
\hline RESUMO \\
\hline 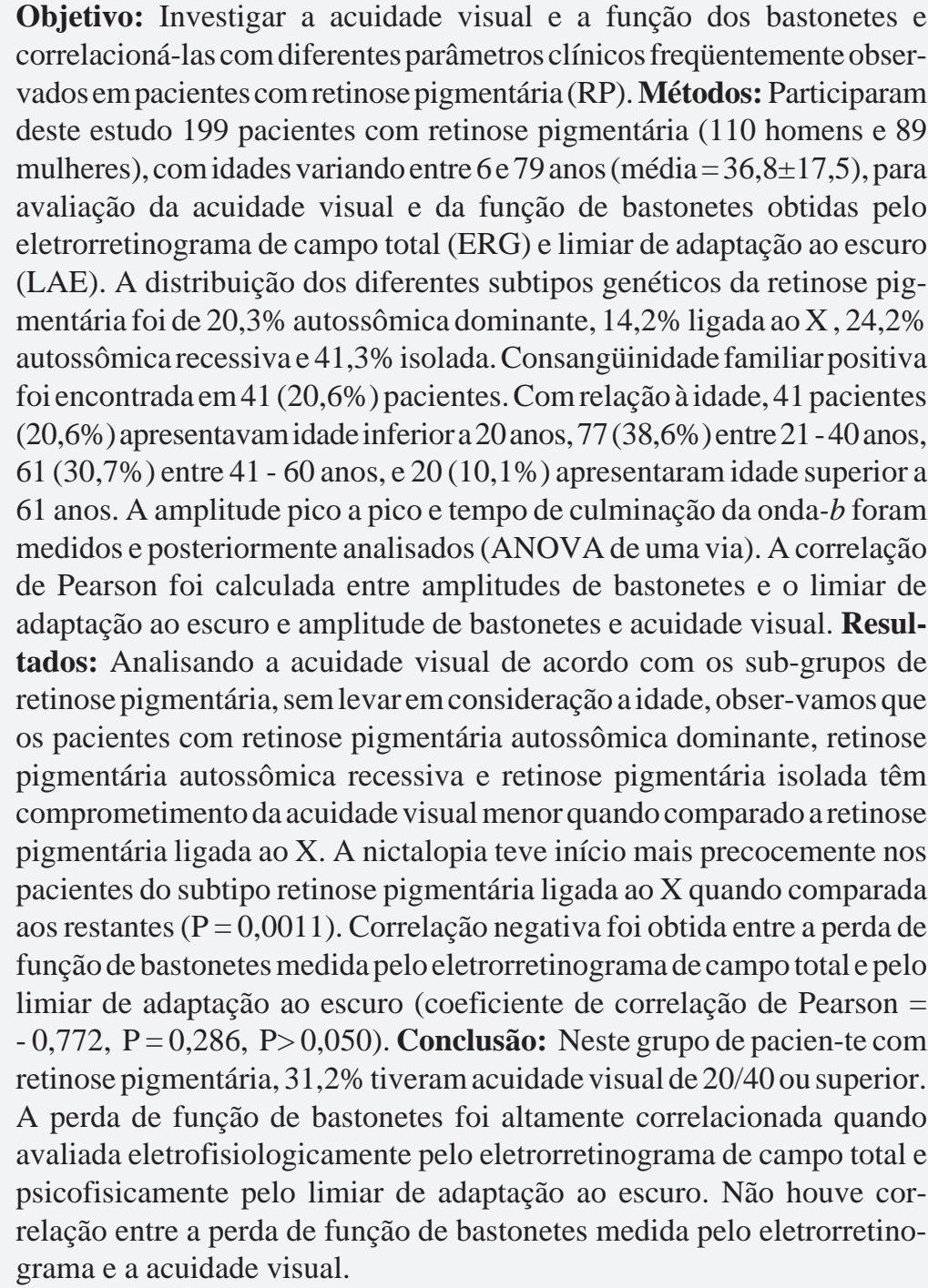 \\
\hline
\end{tabular}

Descritores: Acuidade visual; Campos visuais; Retinite pigmentosa; Bastonetes (Retina); Eletrorretinografia; Adaptação à escuridão; Eletrofisiologia 


\section{INTRODUÇÃO}

Retinose pigmentária (RP) inclui um grupo de distrofias retinianas progressiva hereditária caracterizada por nictalopia, alteração de epitélio pigmentário da retina (EPR), perda progressiva de campo visual periférico e diminuição ou resposta não detectável no exame de eletrorretinograma $(E R G)^{(1-2)}$.

Os sintomas mais comuns são nictalopia, dificuldade de adaptação ao escuro e redução de campo visual periférico. Esta perda de visão periférica pode progredir até restar apenas uma pequena ilha de visão central ${ }^{(3)}$. As características do fundo de olho mais comumente encontradas em pacientes com RP são: atenuação dos vasos da retina presente de $80 \%$ a $90 \%$ dos casos, alteração do EPR e nos estágios mais avançados da doença palidez temporal de papila.

RP pode ser herdada de forma autossômica recessiva, autossômica dominante e ligada ao $\mathrm{X}$, sendo que herança autossômica recessiva é mais comum, seguida pela autossômica dominante. Com o aparecimento da RP em termos de idade é, usualmente, ao fim da primeira década. Na forma recessiva pode começar entre 25 a 35 anos de idade ${ }^{(4)}$.

A severidade da doença está relacionada com a manifestação inicial, taxa de progressão e freqüência das complicações.

$\mathrm{Na}$ literatura, RP não é infreqüente ser citada como "doença causadora de cegueira total" ou referida como causadora de cegueira ${ }^{(1,5)}$.

\section{OBJETIVO}

O objetivo deste estudo é investigar a acuidade visual e a função dos bastonetes, e correlacioná-las aos diferentes parâmetros clínicos freqüentemente observados em pacientes com retinose pigmentária.

\section{MÉTODOS}

Participaram deste estudo 199 pacientes com retinose pigmentária (110 homens e 89 mulheres), com idade variando entre 6 e 79 anos (média $=36,8 \pm 17,5$ ), após a anuência no termo de consentimento livre e esclarecido. A distribuição dos diferentes subtipos genéticos da RP foi de 20,3\% autossômica dominante, 14,2\% ligada ao X, 24,2\% autossômica recessiva e $41,3 \%$ isolada. Consangüinidade familiar positiva foi encontrada em 41 (20,6\%) pacientes. Com relação à idade, 41 pacientes $(20,6 \%)$ apresentavam idade inferior a 20 anos, $77(38,6 \%)$ entre 21 - 40 anos, 61 (30,7\%) entre 41 - 60 anos, e $20(10,1 \%)$ apresentaram idade superior a 61 anos.

\section{PROCEDIMENTO}

\section{Teste de acuidade visual}

A acuidade visual monocular foi obtida com a melhor cor- reção óptica usando a tabela ETDRS (logMAR). O maior valor da acuidade de cada paciente da mais recente visita foi usado posteriormente para análise.

\section{Eletrorretinograma de campo total}

O eletrorretinograma de campo total (ERG) foi realizado em um olho, sorteado ao acaso, após 30 minutos de adaptação ao escuro e dilatação máxima da pupila (diâmetro pupilar mínimo de $6 \mathrm{~mm}$ ), com uma gota de tropicamida a $1 \%$ e uma gota de fenilefrina a $10 \%$. Após a adaptação ao escuro foi instilada uma gota de colírio de tetracaína (anestésico) e um eletrodo de lente de contato bipolar com filamento de ouro (GoldLens electrode, Doran Instruments, MD, USA) foi inserido no olho, com colírio de metilcelulose a $2 \%$ para proteção da superfície da córnea. Um eletrodo terra de cúpula de ouro em forma de clipe foi colocado no lobo da orelha ipsilateral, preenchido com gel eletrolítico. Para realização do ERG foi utilizado o sistema eletrodiagnóstico UTAS E-3000 (LKC Technologies Inc., Gainthersburg, MD, USA) com amplificação de 10.000 vezes, com filtros eletrônicos variando de $0,3 \mathrm{~Hz}$ a $500 \mathrm{~Hz}$. As amplitudes da resposta foram medidas em microvolts $(\mu \mathrm{V})$ e o tempo de culminação em milisegundos (ms).

Nas cinco respostas do protocolo padrão da ISCEV, foram feitas 20 apresentações dos estímulos luminosos, sendo considerada a resposta final a média destes 20 registros, com exceção da resposta de flicker em que foi feita a média de 50 apresentações dos estímulos luminosos.

Os registros foram obtidos na seguinte ordem:

1) Resposta escotópica de bastonetes, com estimulação de uma luz branca com intensidade máxima atenuada em 2,5 unidades logarítmicas por filtro de densidade neutra $\left(0,0098 \mathrm{~cd} / \mathrm{m}^{2}\right)$. O intervalo interestímulos foi de 2 segundos. Este estímulo fica abaixo do limiar de resposta dos cones produzindo apenas respostas de bastonetes.

2) Resposta escotópica máxima, obtida com estímulos de alta intensidade $\left(2,467 \mathrm{~cd} / \mathrm{m}^{2}\right)$. Para esta resposta houve contribuição dos cones e bastonetes. O estímulo é apresentado ainda com o olho adaptado ao escuro e sem a presença de luz de fundo. O intervalo interestímulos foi de 10 segundos, devido à alta intensidade do mesmo.

3) Resposta escotópica de potenciais oscilatórios, com intervalo interestímulos de 15 segundos e filtro de corte a $75 \mathrm{~Hz}$. Os potenciais oscilatórios apresentam dois picos proeminentes aparecendo na porção ascendente da resposta escotópica máxima e refletem a atividade das camadas internas da retina.

4) Resposta fotópica de cones ao flash único, com intervalo interestímulos de 1 segundo, após pré-adaptação por 10 minutos a uma luz de fundo branca com luminância de $30 \mathrm{~cd} / \mathrm{m}^{2}$. Esta resposta contém uma onda- $a$ proeminente com tempo de culminação de aproximadamente $15 \mathrm{~ms}$, uma onda- $b$.

5) Resposta fotópica de flicker a $30 \mathrm{~Hz}$, resposta isolada dos cones com estimulação intermitente a uma freqüência de 
$30 \mathrm{~Hz}$, registrada na presença de uma luz de fundo com luminância de $30 \mathrm{~cd} / \mathrm{m}^{2}$, após um período de adaptação do olho à luz. A amplitude da resposta foi medida pico a pico, ou seja, o tamanho da resposta foi medido do pico da resposta mínima ao pico da resposta máxima. O tempo de culminação foi medido a partir do início do estímulo até o pico máximo da onda- $b$

A amplitude pico a pico e o tempo de culminação da onda$b$ de acordo com o protocolo recomendado pela ISCEV ${ }^{(6)}$ foram comparados valores de normalidade prévios com a mesma técnica empregada ${ }^{(7-8)}$.

\section{Teste de limiar de adaptação ao escuro}

O limiar de adaptação ao escuro (L.A.E.) foi obtido utilizando-se o SST-1 (LKC Technologies). O SST-1 contém um LED campo total verde $\left(\lambda_{\max }=572 \mathrm{~nm}\right)$ com o modo de estímulo em adaptometria, com a intensidade máxima de $-2,15 \log \mathrm{cd} / \mathrm{m}^{2}$ a $30 \mathrm{~dB}$. A intensidade do estímulo SST-1 decresce em intervalos de $1 \mathrm{~dB}$ e aumenta em degraus de $5 \mathrm{~dB}$, variando de 0 a $30 \mathrm{~dB}$. As determinações dos limiares no SST-1 foram obtidas usando um protocolo de escadas de escolha forçada de duas alternativas (estímulo, não estímulo, $n=3$, escadas decrescidas de $1 \mathrm{~dB}$ ). O limiar de adaptação final foi obtido através da mediana entre três determinações ${ }^{(9)}$.

\section{PARÂMETROS}

A amplitude da onda- $a$ é medida da linha de base até o pico negativo, seguido pela amplitude da onda- $b$ até o pico positivo. O tempo de culminação da onda- $b$ foi medido a partir do início do estímulo até seu pico ${ }^{(10)}$. O limiar de adaptação ao escuro foi obtido em $\mathrm{dB}$ e transformado em log (dB) para transformar a distribuição em gaussiana, o mesmo ocorrendo com o valor da amplitude de bastonetes calculada em $\log (\mu \mathrm{V})$ para tornar possíveis os dados comparáveis no cálculo da correlação entre os parâmetros de interesse.

\section{MÉTODO ESTATÍSTICO}

As análises estatísticas foram realizadas através do programa estatístico Jandel Sigmastat-Statistical Software Version 2.0, USA. A amplitude pico a pico e o tempo de culminação da onda- $b$ foram medidas e posteriormente analisadas por análise de variância (ANOVA) de uma via. A correlação de Pearson foi calculada entre a amplitude de bastonetes e o limiar de adaptação ao escuro e amplitude de bastonetes e acuidade visual.

\section{RESULTADOS}

A figura 1 mostra um exemplo representativo do ERG de campo total (resposta escotópica de bastonetes) de um paciente do subtipo da RP ligada ao X com 27 anos e a acuidade

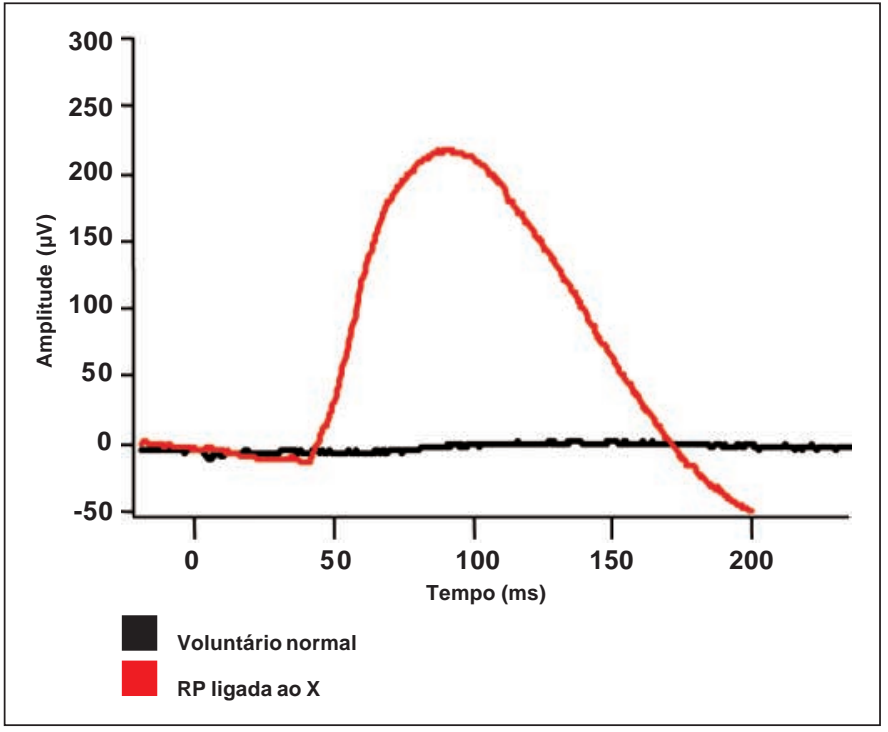

Figura 1 - Registro representativo da resposta escotópica de bastonetes de um voluntário normal, 27 anos e acuidade visual de 20/20 e um paciente com RP ligada ao X, 27 anos e acuidade visual 20/20

visual 20/20, comparando com um voluntário normal de idade similar e mesma acuidade.

Analisando a acuidade visual de acordo com os subgrupos de RP, sem levar em consideração a idade, observamos que os pacientes com RP autossômica dominante, RP autossômica recessiva e RP isolada têm um comprometimento da acuidade visual menor quando comparado a RP ligada ao X, visto que $58 \%$ dos pacientes com RP ligada ao X poderiam ser considerados legalmente cegos caso adotássemos apenas o critério da acuidade visual (Tabela 1).

A figura 2 mostra a correlação negativa da perda de função dos bastonetes medida pelo ERG e pelo L.A.E. (coeficiente de correlação de Pearson= - 0,772, $\mathrm{P}=0,000)$.

Não houve correlação entre a acuidade visual e a perda de função de bastonetes (coeficiente de correlação de Pearson = $0,0815, \mathrm{P}=0,286, \mathrm{P}>0,050$ ), como mostra a figura 3 .

A amplitude de bastonetes e o tempo de culminação da onda- $b$ nos quatro subtipos de RP estudados foram comparáveis $(\mathrm{P}=0,366$ e $\mathrm{P}=0,446$ respectivamente $)$.

\section{DISCUSSÃO}

$\mathrm{Na}$ literatura, a RP é freqüentemente referida como uma doença que causa cegueira ${ }^{(11-12)}$, porém muitas destas citações não especificam a definição de cegueira, a qual pode se referir: a cegueira total ou a cegueira legal. Considera-se cegueira legal de acordo com o critério da acuidade visual quando com a melhor correção óptica a acuidade visual não é melhor que 20/200 no melhor olho e/ou campo visual inferior a $10^{\circ}$. Neste estudo 69 (34,6\%) dos pacientes seriam considerados legalmente cegos levando-se em conta apenas o critério da baixa de acuidade visual. Vinte e oito $(70 \%)$ dos pacientes do subtipo 


\begin{tabular}{|c|c|c|c|c|c|}
\hline AV & RP A. Dom & RP XL & RP A. Rec & RP Iso & Total \\
\hline $20 / 40$ ou melhor & 11 (28\%) & $6(21 \%)$ & $18(37 \%)$ & 27 (33\%) & 62 \\
\hline $20 / 50-20 / 150$ & 17 (42\%) & $6(21 \%)$ & 17 (35\%) & $29(35 \%)$ & 69 \\
\hline $20 / 200-20 / 400$ & $4(10 \%)$ & $9(33 \%)$ & $3(6 \%)$ & $8(10 \%)$ & 24 \\
\hline $20 / 400$ ou pior & $8(20 \%)$ & $7(25 \%)$ & 11 (22\%) & $18(22 \%)$ & 44 \\
\hline Num. de casos & 40 & 28 & 49 & 82 & 199 \\
\hline
\end{tabular}

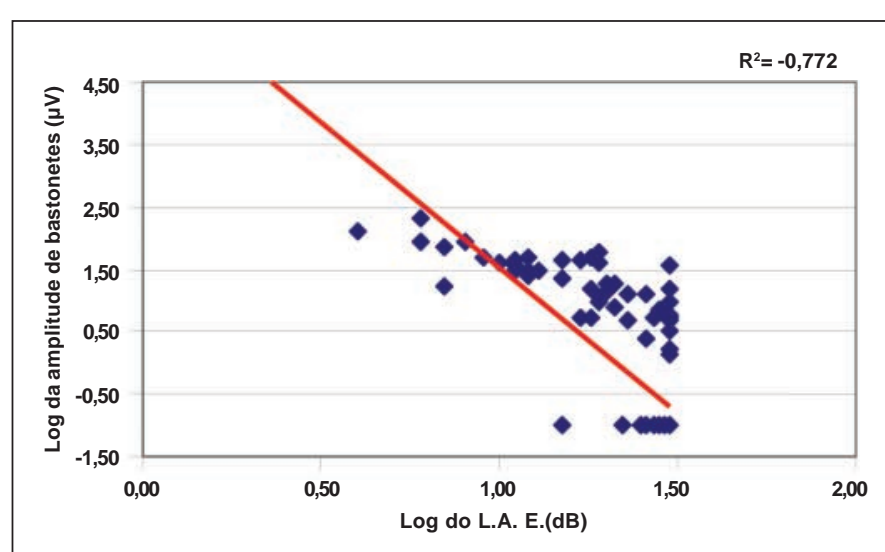

Figura 2 - Limiar de adaptação ao escuro e resposta escotópica de bastonetes

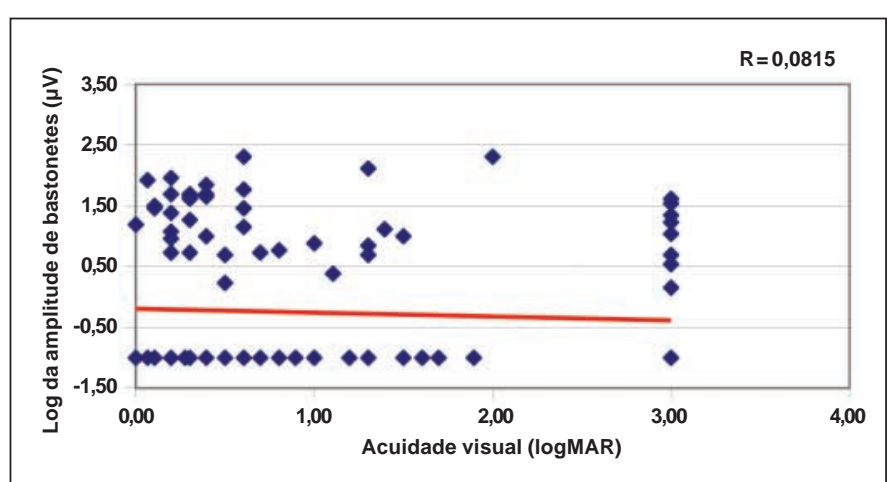

Figura 3 - Acuidade visual e resposta escotópica de bastonetes

de RP autossômica dominante obtiveram acuidade visual de 20/150, ou melhor, e 12 (42\%) na RP ligada ao X. Num estudo com 58 pacientes com RP, a acuidade visual média de $0,35 \mathrm{log}$ MAR (equivalente de Snellen 20/45), independentemente da hereditariedade ${ }^{(13)}$. No nosso estudo encontramos em $31 \%$ a acuidade visual média melhor do que 20/40, sem levar em consideração o tipo de RP. Os valores de acuidade visual encontrados neste estudo vão de acordo com os previamente descritos na literatura ${ }^{(14-16)}$.

A perda de função de bastonetes foi altamente correlacionada quando avaliada eletrofisiologicamente (ERG) e psicofisicamente (LAE), reafirmando dados anteriormente descri$\operatorname{tos}^{(9,17)}$. Não houve correlação entre a perda de função de bastonetes medida através do ERG e a acuidade visual, mostrando que $31,2 \%$ dos pacientes com RP tiveram ERG extinto e acuidade visual de 20/40 ou superior como era esperado, pois o ERG avalia a função da retina periférica e médio-periférica e a acuidade visual é uma medida da função retiniana macular. $\mathrm{O}$ curso da evolução da RP é afetar inicialmente a periferia da retina, e continuar em direção a área central ${ }^{(3)}$. A função retiniana medida pelo eletrorretinograma de campo total e o limiar de adaptação ao escuro pode ser muito útil na determinação da progressividade da RP.

O limiar de adaptação ao escuro é um exame rápido, não invasivo, quantitativo e de custo relativamente baixo quando comparado ao ERG. Porém por se tratar de um exame subjetivo, que exige a colaboração e o entendimento do exame por parte do paciente os resultados não são tão precisos em relação ao ERG. O ERG contribui na avaliação objetiva e quantitativa da função retiniana com resultados precisos e confiáveis, porém o equipamento é caro e exige um examinador extremamente treinado para aquisição e análise posterior dos dados.

\section{CONCLUSÃO}

Neste grupo de paciente com retinose pigmentária, 31,2\% tiveram acuidade visual de 20/40 ou superior. A perda de função de bastonetes foi altamente correlacionadas quando avaliadas eletrofisiologicamente pelo eletrorretinograma de campo total e psicofisicamente pelo limiar de adaptação ao escuro. Não houve correlação entre a perda de função de bastonetes medida pelo eletrorretinograma e a acuidade visual.

\section{ABSTRACT}

Purpose: To investigate visual acuity and rod function, and correlate them to different clinical parameters in patients with retinitis pigmentosa (RP). Methods: A cohort of 199 patients with retinitis pigmentosa (110 males and 89 females), aged 679 years (mean $=36.8 \pm 17.5$ ) had their monocular visual acuity measured by the ETDRS chart and rod function assessed by full-field electroretinogram and dark-adapted thresholds. The distribution of different genetic subtypes of retinitis pigmentosa was $20.3 \%$ autosomal dominant, $14.2 \% \mathrm{X}$ - linked, $24.2 \%$ autosomal recessive and $41.3 \%$ isolated. History of consanguinity was found in $41(20.6 \%)$ patients. Forty-one patients 
(20.6\%) were 20 years old or less, $77(38.6 \%)$ ranged from 21 40 years, $61(30.7 \%)$ from 41 - 60 years, and $20(10.1 \%)$ were 61 years or older. Peak-to-peak amplitude and $b$-wave implicit time were measured and statistically analyzed (one-way ANOVA). Pearson correlation was performed between rod amplitude and dark-adapted thereshold and rod amplitude and visual acuity. Results: Analyzing the visual acuity data according to genetic subtypes, without considering age, showed that as a group, patients with autosomal recessive and isolated retinitis pigmentosa have less severe impairment of visual acuity, than those with X-linked retinitis pigmentosa. Nyctalopia begun earlier in $\mathrm{X}$-linked groups, compared with the remaining groups $(\mathrm{p}=0.011)$. A negative correlation was found between dark-adapted thereshold and scotopic rod amplitude (Pearson correlation coefficient $=-0.772$ and $\mathrm{P}=0.000$ ). There were no significant relationships between visual acuity and rod response by electroretinogram (Pearson correlation coefficient $=0.0815$ and $P=0.286), P>0.050$. Conclusions: In a cohort of retinitis pigmentosa patients, $31.2 \%$ had vision of $20 / 40$ or better. Rod function loss was highly correlated when assessed electrophysiologically (ERG) and psychophysically (dark-adapted thershold). No correlation was found between rod response measured by electroretinogram and visual acuity.

Keywords: Visual acuity; Visual fields; Retinitis pigmentosa; Rods (Retina); Electroretinography; Dark adaptation; Electrophysiology

\section{REFERÊNCIAS}

1. Grover S, Fishman GA, Anderson RJ, Tozatti MS, Heckenlively JR, Weleber RG, et al. Visual acuity impairment in patients with retinitis pigmentosa at 45 years older. Ophthalmology. 1999;106(9):1780-5.
2. Weleber RE. Retinitis pigmentosa and allied diseases. In: Ryan SJ, editor. Retina. 2a ed. Louis: Mosby; 1994. p.335-466.

3. Allard RE. Retinitis pigmentosa - An overview. J Am Optom Assoc. 1983; 54(9):793-800.

4. Kril AE. Retinitis pigmentosa: a review. Sight Saving Rev. 1972;42(1):21-8.

5. Grover S, Fishman GA, Alexander KR, Anderson RJ, Derlacki DJ. Visual acuity impairment in patients with retinitis pigmentosa. Ophthalmology. 1996;103(10):1593-600.

6. Marmor MF, Zrenner E. Standard for clinical electroretinography (1999 update). International Society for Clinical Electrophysiology of Vision. Doc Ophthalmol. 1998-99;97(2):143-56.

7. Birch DG, Anderson JL. Standardized full field electroretinography. Normal values and their variation with age. Arch Ophthalmol. 1992;110(11):1571-6.

8. Pereira JM, Mendieta L, Sacai PY, Salomão SR, Berezovsky A. Estudo normativo do eletrorretinograma de campo total em adultos jovens. Arq Bras Oftalmol. 2003;66(2):137-44.

9. Peters AY, Locke KG, Birch DG. Comparision of de Goldmann-Weekers dark adaptometer and LKC Technologies scotopic sensitivity tester - 1. Doc Ophthalmol. 2000;101(1):1-9.

10. Flores-Guevara R, Renault F, Ostré C, Richard P. Maturation of the electroretinogram in children: estabilish of the amplitude ratio a/b. Electroencephalogr Clin Neurophysiol, 1996; 100(5): 422-7.

11. Friederich R. Eye disease in the navajo indians. Ann Ophthalmol. 1982;14 (1):38-40.

12. Bloome MA, Garcia CA. Manual of retinal and choroidal dystrophies. New York: Appleton-Century-Crofts; 1982.

13. Paranhos FRL, Nehemy MB, Hirose T. Bilateralidade, simetria e correlação clínico-eletrofisiológico na retinose pigmentar. Arq Bras Oftalmol. 1998;61 (4):386-9.

14. Pearlman JT. Mathematical models of retinites pigmentosa: study of the rate of progress in defferent genetic forms. Trans Am ophthalmol Soc. 1979;77: 643-56

15. Berson EL, Sandberg MA, Rosner B, Birch DG, Hanson AH. Natural course of retinitis pigmentosa over a three-year interval. Am J Ophthalmol. 1985;99 (3):240-51.

16. Paranhos FRL, Katsumi O, Arai M, Nehemy MB, Hirose T. Pattern reversal visual evoked response in retinitis pigmentosa. Doc Ophthalmol. 1998-99;96 (4):321-31

17. Birch DG, Fish GE. Rod ERGs in retinitis pigmentosa and cone-rod degeneration. Invest Ophthalmol Vis Sci. 1987;28(1):140-50.

\section{CONGRESSO PAN-AMERICANO DE OFTALMOLOGIA}

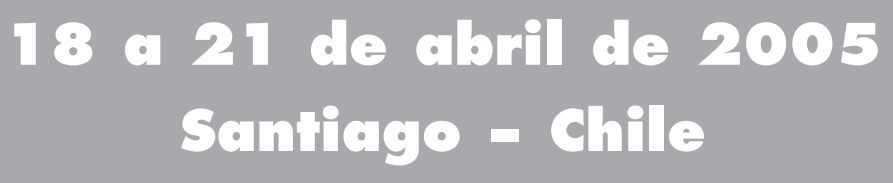

INFORMAC̣ÕES: E-mail: info@paao.org 